\title{
Dissecting the interaction between tissue inhibitor of metalloproteinases-3 (TIMP-3) and low density lipoprotein receptor-related protein-1 (LRP-1): development of a "TRAP" to increase levels of TIMP-3 in the tissue
}

\author{
Simone D. Scilabraa,c,d, Kazuhiro Yamamoto ${ }^{\mathrm{b}}$, Martina Pigoni ${ }^{\mathrm{c}}$, Kazuma Sakamoto ${ }^{\mathrm{a}}$, \\ Stephan A. Müller ${ }^{c}$, Alkmini Papadopoulou ${ }^{c}$, Stefan F. Lichtenthaler, ${ }^{c, d, e}$, Linda \\ Troeberg $^{b}$, Hideaki Nagase ${ }^{b}$, Kenji Kadomatsu ${ }^{a}$ \\ a- Department of Biochemistry, Nagoya University Graduate School of Medicine, Nagoya, \\ Japan \\ b- Kennedy Institute of Rheumatology, Nuffield Department of Orthopaedics, Rheumatology \\ and Musculoskeletal Sciences, University of Oxford, Oxford, United Kingdom \\ c- Department of Neuroproteomics, Deutsches Zentrum für Neurodegenerative \\ Erkrankungen (DZNE), Munich, Germany \\ d- Neuroproteomics, Klinikum rechts der Isar and Institute for Advanced Study, Technische \\ Universität München, Munich, Germany; \\ e- Munich Cluster for Systems Neurology (SyNergy), Ludwig-Maximilians-University, Munich, \\ Germany.
}

Correspondence to: Dr. Simone D. Scilabra, Department of Neuroproteomics, Deutsches Zentrum für Neurodegenerative Erkrankungen (DZNE) München, Feodor-Lynen-Strasse 17, 81377 Munich, Germany; Telephone: +49 (0) 89 4400-46436; FAX: +49 (0) 89 4400-46429; E-mail: simone.scilabra@dzne.de

\begin{abstract}
Tissue inhibitor of metalloproteinases 3 (TIMP-3) is a key regulator of extracellular matrix turnover for its ability to inhibit matrix metalloproteinases (MMPs), adamalysin-like metalloproteinases (ADAMs) and ADAMs with thrombospondin motifs (ADAMTSs). TIMP-3 is a secreted protein whose extracellular levels are regulated by endocytosis via the lowdensity-lipoprotein receptor-related protein-1 (LRP-1). In this study we developed a molecule able to "trap" TIMP-3 extracellularly, thereby increasing its tissue bioavailability. LRP-1 contains four ligand-binding clusters. In order to investigate the TIMP-3 binding site on LRP1 , we generated soluble minireceptors (sLRPs) containing the four distinct binding clusters or part of each cluster. We used an array of biochemical methods to investigate the binding of TIMP-3 to different SLRPs. We found that TIMP-3 binds to the ligand-binding cluster II of the receptor with the highest affinity and a soluble minireceptor containing the N-terminal half of cluster II specifically blocked TIMP-3 internalization, without affecting the turnover of metalloproteinases. Mass spectrometry-based secretome analysis showed that this minireceptor, named T3TRAP, selectively increased TIMP-3 levels in the extracellular space and inhibited constitutive shedding of a number of cell surface proteins. In conclusion, T3TRAP represents a biological tool that can be used to modulate TIMP-3 levels in the tissue and could be potentially developed as a therapy for diseases characterized by a deficit of TIMP-3, including arthritis.
\end{abstract}

\section{INTRODUCTION}

The timely degradation of extracellular matrix (ECM) components is crucial for several biological processes, including cell migration, development and tissue repair (1). ECM turnover involves the activity of different classes of proteinases, with the matrix metalloproteinases (MMPs) and adamalysin-like metalloproteinases with thrombospondin domains (ADAMTSs) playing a leading role among them. The related family of membrane-anchored adamalysins (ADAMs) proteolytically cleave proteins at the cell surface, thereby controlling the communication between cells and their environment (2). The activity of MMPs, ADAMTSs and ADAMs is physiologically regulated through formation 
of a 1:1 stochiometric complex with their endogenous inhibitors called tissue inhibitors of metalloproteinases (TIMPs) (3), which comprise four members in mammals. Among them, TIMP-3 has a broader inhibitory profile, being unique in its ability to inhibit members of all three of these classes of metalloproteinases and regulate their activity in vivo (4). Timp3-null mouse displays a number of defects associated with excess of extracellular matrix (ECM) turnover, including signs of premature arthritis due to elevated activity of MMPs and ADAMTSs (4-6). Moreover, the Timp3null mouse releases high levels of TNFa upon challenge with surgical resection of the liver and other inflammatory models, due to excess ADAM17 activity (7). Decreased levels of TIMP-3 are present in degraded human osteoarthritis (OA) cartilage compared to normal cartilage. On the other hand, an increase in TIMP-3 levels has been associated with protective inhibition of cartilage degradation in arthritis. For example, addition of TIMP-3 to cartilage explants has been shown to inhibit ADAMTS-mediated aggrecan degradation (8) and intra-articular injection of TIMP-3 inhibited cartilage breakdown in a rat model of OA (9). For this reason, strategies to increase levels of TIMP-3 in the tissue have been widely sought as potential therapeutic treatment for arthritis and other conditions associated with deficit of the inhibitor.

We previously discovered that extracellular levels of TIMP-3 are regulated by low-density-lipoprotein receptor-related protein-1 (LRP-1)-mediated endocytosis $(10,11)$. As a consequence, no or little amount of TIMP-3 accumulates in the extracellular milieu unless its endocytosis is blocked (11). LRP-1 is a multidomain endocytic receptor that belongs to the LDL receptor family and is known to internalize more than 50 ligands through four ligandbinding clusters (12). LRP-1 can undergo proteolytic shedding from the cell surface, with the subsequent release of a soluble form of the receptor (sLRP-1). We found that SLRP-1 competed with cell-surface LRP-1 for binding to TIMP-3 and increased its extracellular levels (10). Furthermore, when TIMP-3 is bound to SLRP-1, it retains its inhibitory activity against metalloproteinases, suggesting that shedding of LRP-1 and subsequent formation of sLRP-1/TIMP-3 complexes is a physiological mechanism to increase TIMP3 bioavailability in the extracellular milieu (10).

In this study, we identified the major binding site for TIMP-3 on LRP-1 in the ligand-binding cluster II. Additionally, we found that a soluble minireceptor containing the N-terminal half of cluster II specifically binds to TIMP-3, but not its target metalloproteinases, and it selectively traps the inhibitor in the extracellular milieu. Unbiased shot-gun proteomics showed that this minireceptor, that we named T3TRAP, was able to inhibit constitutive shedding of a number of cell surface proteins, including the leukocyte surface antigen CD47, integrin beta-1 and cell adhesion molecule1. In conclusion, T3TRAP represents an effective biological tool to modulate TIMP-3 levels in the extracellular space without affecting metalloproteinase turnover, thereby inhibiting proteolysis in the tissue. Potentially, it can prove beneficial in diseases characterized by deficit of TIMP-3, including cancer, arthritis and atherosclerosis.

\section{RESULTS}

\subsection{Mapping of TIMP-3 binding sites on LRP-1}

LRP-1 is an endocytic receptor with a characteristic modular structure, with four ligand-binding clusters (LRPI-IV) driving the interaction between the receptor and its ligands. Each cluster contains sequences of 2, 8, 10 and 11 cysteine-rich complementtype repeats (CRs), respectively. All clusters comprise two EGF-like repeats at the Cterminus, while domain II, III and IV also contain one EGF-like repeat at the $\mathrm{N}$ terminus (Figure 1A). To test which ligandbinding cluster of LRP-1 interacts with TIMP-3, four soluble minireceptors containing the different ligand-binding clusters (sLRP1-4) were purified and their binding to TIMP-3 was measured by ELISA. TIMP-3 bound to SLRP2 with the greatest affinity, showing a $K_{D, a p p}$ of $7 \mathrm{nM}$ (Figure 1B), similar to the affinity of TIMP-3 for full length LRP-1 in this assay ( $K_{D, \text { app }}$ of $\left.10 \mathrm{nM}\right)$. TIMP3 bound to SLRP1, SLRP3 and SLRP4, but with lower affinity. 


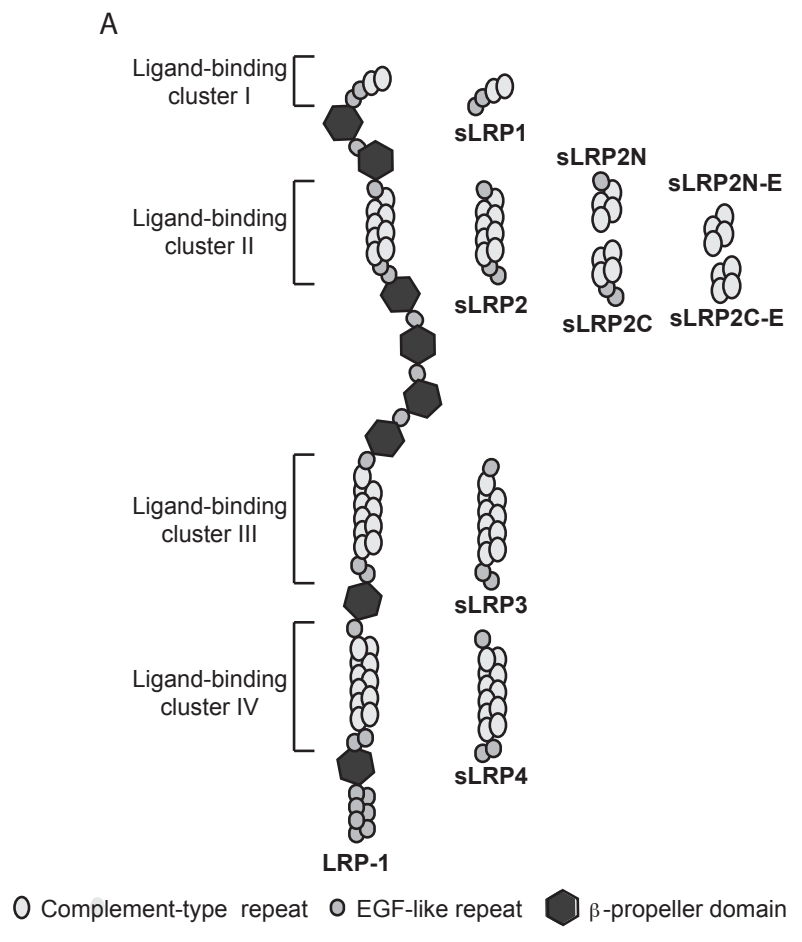

B
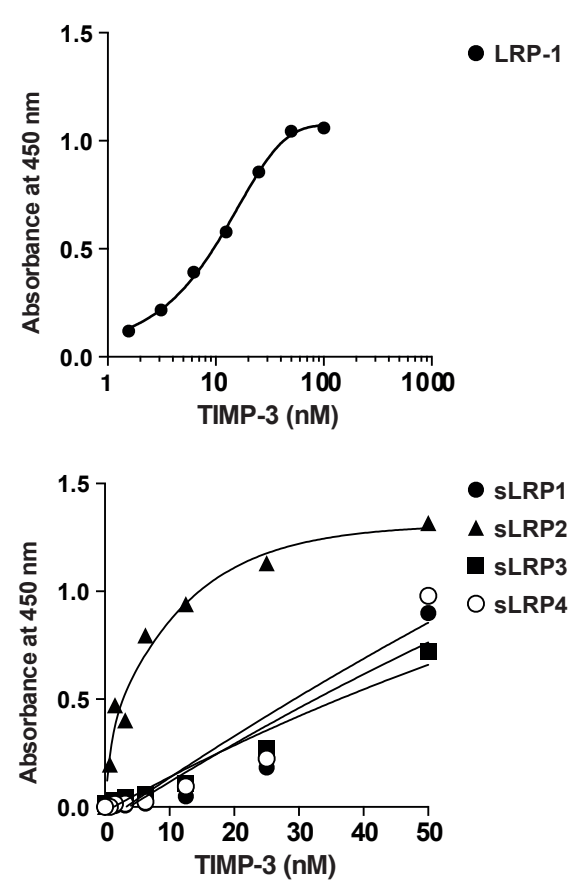

Figure 1. Binding of TIMP-3 to ligand-binding clusters of LRP-1. (A) Schematic representation of various SLRP minireceptors used in this study. (B) Full-length LRP-1 or recombinant LRP-1 ligand-binding clusters I (sLRP1), II (sLRP2), III (sLRP3) and IV (sLRP4) were coated onto microtiter plates and binding of TIMP-3-FLAG (0-100 nM) was measured using an M2 anti-FLAG antibody and a horseradish peroxidase-conjugated secondary antibody

\subsection{Development of a T3TRAP that increases extracellular bioavailability of TIMP-3.}

In order to develop a "trap" that would specifically increase extracellular levels of TIMP-3 (T3TRAP), we aimed to identify a fragment of LRP-1 that interacts with TIMP-3 and promotes its extracellular accumulation, without affecting the LRP-1mediated endocytosis of its target metalloproteinases. Having TIMP-3 shown the highest affinity for sLRP2 among the four binding domains, we investigated whether minireceptors consisting of the $\mathrm{N}$ terminal or C-terminal half of sLRP2 (termed sLRP2N and sLRP2C) were able to accumulate TIMP-3 in the medium of cutured U251 glioblastoma cells. Serial dilutions of conditioned media containing standardized amount of each minireceptor were added to U251 glioblastoma cells and
TIMP-3 accumulation was evaluated. Figure $2 \mathrm{~A}$ and $\mathrm{B}$ shows that sLRP2N dosedependently increased accumulation of TIMP-3 in the conditioned media. A minireceptor consisting of SLRP2 without the EGF-like repeat (sLRP2N-E), was able to accumulate TIMP-3 in the medium to the same extent of SLRP2N, while the Cterminal half of ligand-binding cluster II (sLRP2C) or sLRP2C without EGF-like repeats (sLRP2C-E) did not induce extracellular accumulation of the inhibitor. Together with previous results showing that TIMP-3 binds to sLRP2 with the highest affinity, these results indicate that the major TIMP-3 binding site resides on the $\mathrm{N}$ terminal moiety of LRP-1 binding cluster II, and that EGF-like repeats do not play a major role in this interaction. As a result, SLRP2N-E was considered as a possible T3TRAP and further investigated. We had 
previously shown that TIMP-3 endocytosis was inhibited when the protein is in complex with soluble LRP-1(10). Thus, we confirmed that the accumulation of TIMP-3 in the presence of SLRP2N-E was due to the inhibition of its endocytosis. Indeed, the endocytosis of TIMP-3 slows down when the inhibitor is in complex with SLRP2N-E
(Figure 2C). Then, we tested the ability of SLRP2N-E to increase TIMP-3 in the extracellular milieu of cells other than U251. Similarly to U251 cells, the presence of sLRP2N-E induced a dose-dependent accumulation of TIMP-3 in the conditioned media of COS1 and PC3 cells (Figure 2D).
A

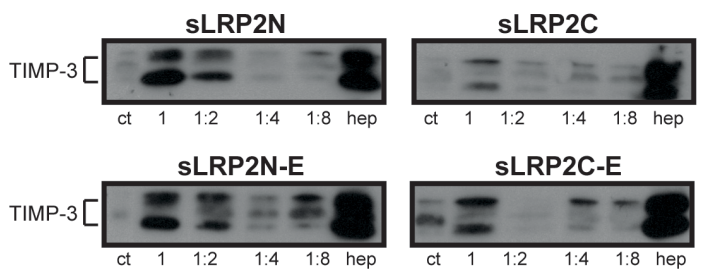

C
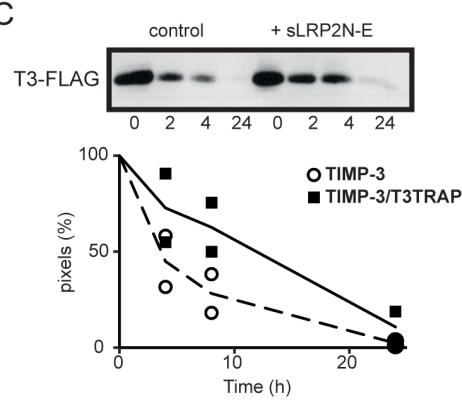

B

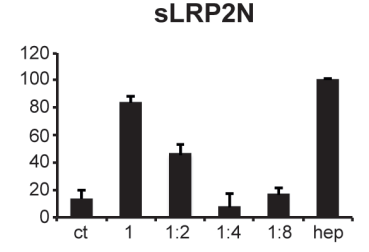

SLRP2N-E
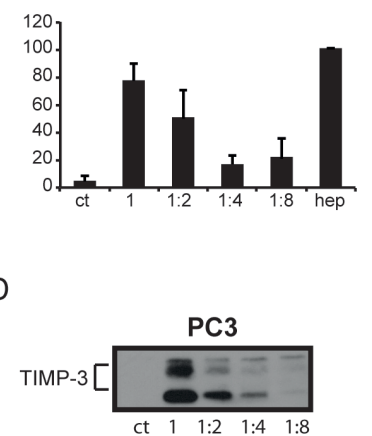

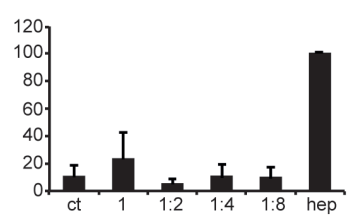

sLRP2C

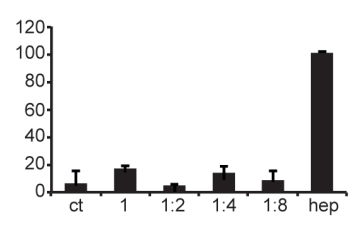

SLRP2C-E

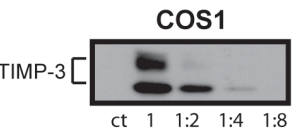

Figure 2. sLRP2N-E induces accumulation of TIMP-3 in conditioned media. (A) U251 cells were incubated for $24 \mathrm{~h}$ with serial dilutions of conditioned media containing distinct sLRP minireceptors, conditioned media from mock transfected cells (ct) or conditioned media from mock transfected cells containing $200 \mu \mathrm{g} / \mathrm{ml}$ heparin (hep). The amount of TIMP3 accumulated in the extracellular space was analyzed by immunoblotting. (B) Bands corresponding to accumulated TIMP-3 were quantified using ImageJ and standardized with values of heparin for each blot $(n=3)$. (C) FLAG-tagged TIMP-3 (5 nM) either alone or in the presence of SLRP2N-E was added to U251 cells. At indicated time points, the amount of TIMP-3-FLAG in the conditioned media was evaluated by immunoblotting using an antiFLAG M2 antibody. The intensity of bands corresponding to TIMP-3-FLAG at different times was quantified and plotted as percentage of values at $0 \mathrm{~h}$ (the experiment was performed twice with similar results). The solid line represents the average values of TIMP-3-FLAG in the presence of SLRP2N-E, while the dashed line represents the values of TIMP-3-FLAG alone. (D) TIMP-3 accumulation in the extracellular milieu of PC3 and COS1 cells was tested in the presence of serial dilutions of SLRP2N-E conditioned medium. 
A
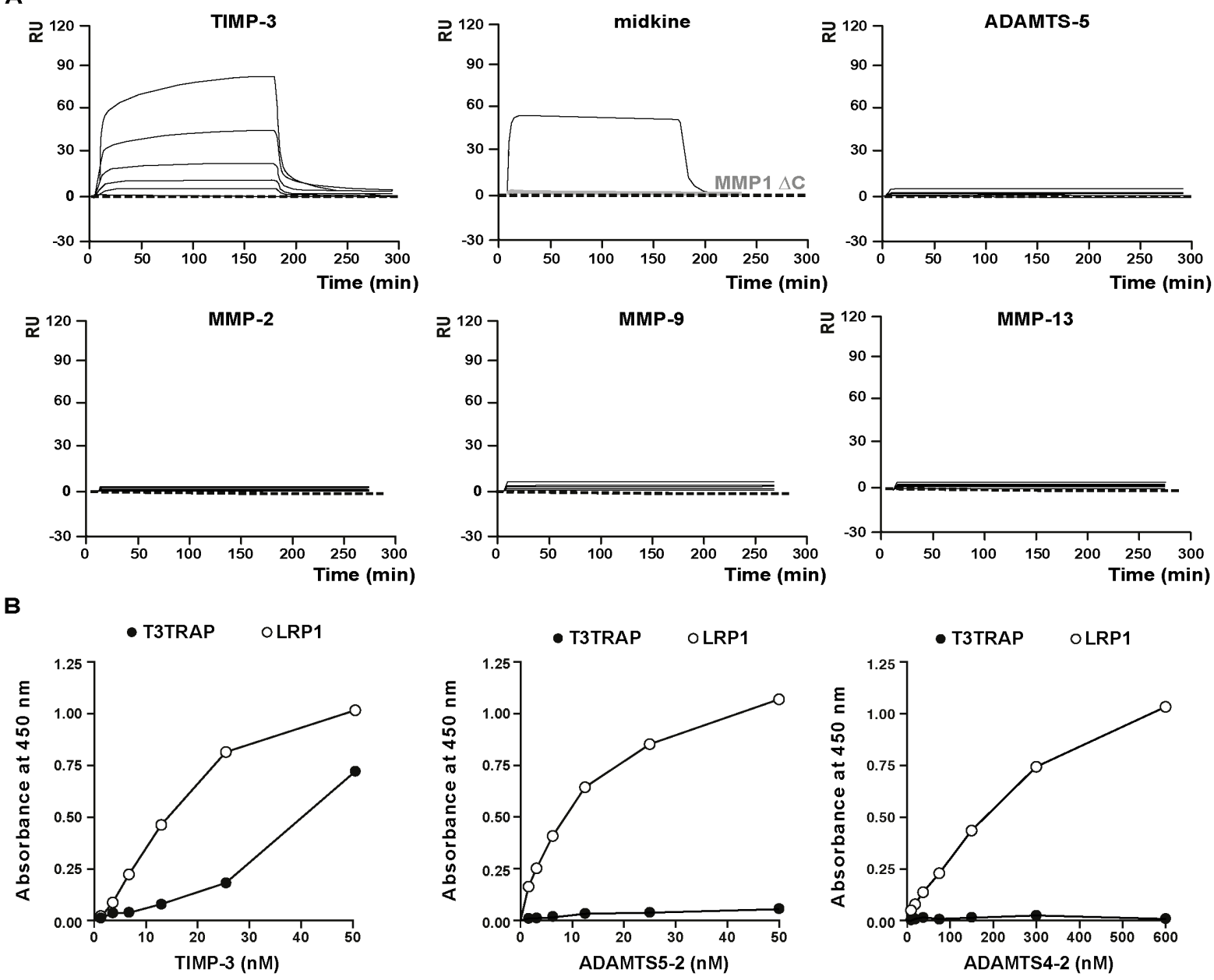

Figure 3. sLRP2N-E is specific for TIMP-3, but not metalloproteinases. (A) Sensograms showing the binding of TIMP-3 $(0-80 \mathrm{nM})$, midkine $(140 \mathrm{nM}), \mathrm{MMP}^{-1} \Delta \mathrm{C}(600 \mathrm{nM})$, ADAMTS-5 (0-80 nM), MMP-2 (0-80 nM), MMP-9 (0-80 nM) or MMP-13 (0-80 nM) to SLRP2N-E. (B) Purified SLRP2N-E or full length LRP-1 were coated onto microtiter plates and binding of FLAG-tagged TIMP-3, ADAMTS5-2 or ADAMTS4-2 was evaluated by ELISA using an M2 anti-FLAG antibody.

\section{3 sLRP2N-E binds to TIMP-3, but not metalloproteinases}

A number of metalloproteinases have been identified as LRP-1 ligands (1316). Therefore, in order to understand the specificity of sLRP2N-E as a T3TRAP we investigated its interaction with these metalloproteinases by SPR. As showed by the sensograms in Figure 3A, TIMP-3 bound to SLRP2N-E at concentrations as low as 5 nM. Midkine, that was previously shown to bind to the same fragment (17), efficiently bound to sLRP2N-E in this assay, while the catalytic domain of MMP-1 that has no affinity for the full-length receptor did not interact with sLRP2N-E at concentrations up to $640 \mathrm{nM}$. In the same assay, recombinant proMMP-2, proMMP-9, proMMP-13 and ADAMTS-5 displayed no affinity for sLRP2N-E (Figure 3A, Table 1). We also tested the selectivity of SLRP2N-E for TIMP3 by ELISA. While TIMP-3-FLAG efficiently bound to sLRP2N-E, neither FLAG-tagged ADAMTS-5 or ADAMTS-4 interacted with the minireceptor, even though their binding to full length LRP-1 was conserved in this assay (Figure 3B, Table 1). These data confirmed that SLRP2N-E is able to function as a selective T3TRAP (summarized in Table 1). 
Table 1. BINDING OF TIMP-3 AND METALLOPROTEINASES TO T3TRAP

Binding of TIMP-3 and selected metalloproteinases to SLRP2N-E (T3TRAP), evaluated by different assays.

\begin{tabular}{lcc} 
Ligand & Assay & Binding \\
\hline TIMP-3 & SPR/ELISA & Positive binding in the nanomolar range \\
& SPR & No binding up to $80 \mathrm{nM}$ \\
ProMMP-2 & SPR & No binding up to $80 \mathrm{nM}$ \\
proMMP-9 & SPR & No binding up to $80 \mathrm{nM}$ \\
proMMP-13 & SPR & No binding up to $80 \mathrm{nM}$ \\
ADAMTS-5 & ELISA & No binding up to $50 \mathrm{nM}$ \\
ADAMTS5-2 & ELISA & No binding up to $600 \mathrm{nM}$ \\
ADAMTS4-2 & & \\
\hline
\end{tabular}

2.4 T3TRAP increases TIMP-3 levels in the conditioned medium and inhibits protein shedding

LRP-1 is known to internalize more than 50 ligands, therefore we investigated which proteins, other than TIMP-3, could be upregulated in the extracellular milieu in the presence of T3TRAP. We performed a mass spectrometry-based secretome analysis of HEK293 cells expressing T3TRAP. This analysis identified 341 secreted proteins and 168 membrane proteins in the conditioned media of both controls and T3TRAP expressing cells (Uniprot annotation). T3TRAP was only detected in transfected cells (average LFQ value $1,58 \mathrm{E}+08$, Table $\mathrm{S} 1$ ). The large majority of proteins detected in the secretome were not regulated by the overexpression of T3TRAP (Figure 4A, Table S1). TIMP-3 levels increased 4.3-fold in the presence of T3TRAP, with the highest degree of statistical significance among all the proteins regulated (Figure 4A and 4B, Table 2). Similarly to TIMP-3, the Chitinase domain-containing protein 1 (CHID1) was found upregulated by the presence of T3TRAP (8.7-fold). Other proteins, including collagen a1(XIV) chain, peroxiredoxin-4, laminin subunit b2 and cholinesterase, were found to be upregulated in the secretome of T3TRAP cells, but with lower relevance compared to TIMP-3 (Figure 4A, Table 2).
Midkine, whose endocytosis had been previously found to be inhibited by SLRP2N$\mathrm{E}$, slightly accumulated in the conditioned media of T3TRAP-expressing cells (1.2-fold, p-value: 0.0027; Table S1) (17).

Some metalloproteinases were detected in the secretome of T3TRAP expressing cells, including ADAMTS-1, ADAMTS-3 and MMP-11, but they were not significantly regulated by the presence of the trap. Similarly, TIMP-1 and TIMP-2, were not regulated by the expression of T3TRAP (Figure 4A and 4B). Interestingly, levels of MMP-2, which is known to be an LRP-1 ligand, were decreased in T3TRAP expressing cells (Figure 4A, Table 2). Similarly to MMP-2, other proteins including insulin-like growth factor II, apolipoprotein E, transforming growth factor beta-1 and agrin, were downregulated in the presence of T3TRAP.

A number of membrane-anchored proteins that are known to be shed by metalloproteinases, including leukocyte surface antigen CD47 (18), integrin beta-1 (19), cell adhesion molecule 1 (20) and CD166 antigen (21), were significantly reduced in the secretome of T3TRAP expressing cells, suggesting that T3TRAP, and subsequent increased levels of TIMP-3, overall inhibited the constitutive metalloproteinase-dependent shedding of membrane proteins (Table 2). 
A

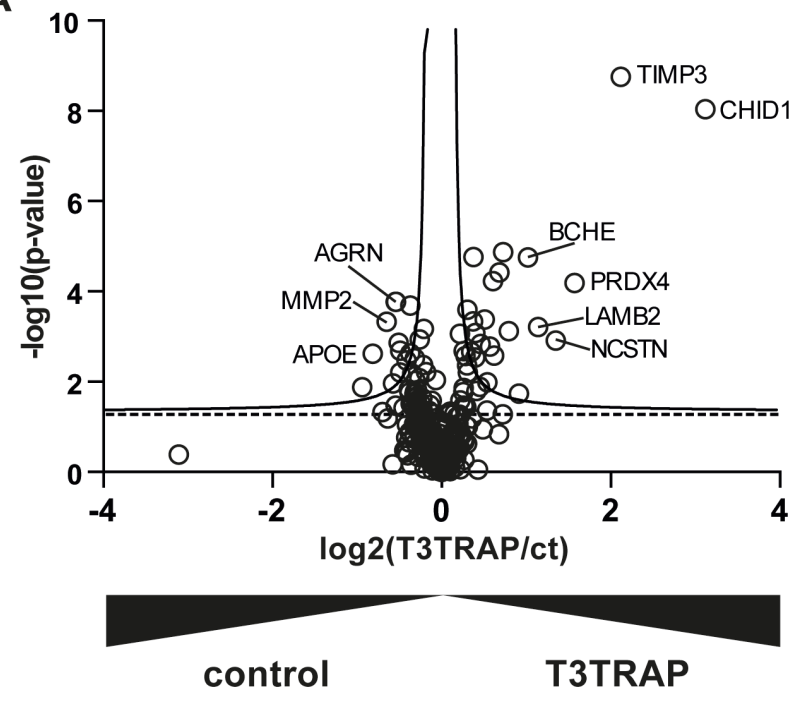

B

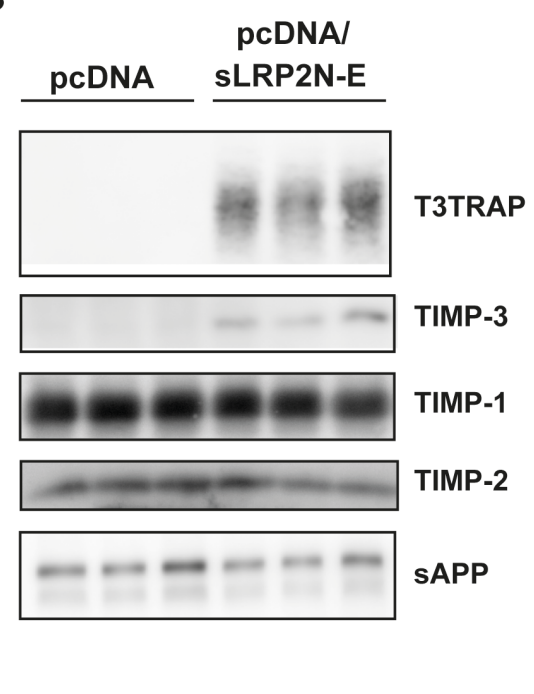

Figure 4. sLRP2N-E selectively increases TIMP-3 extracellularly. (A) Volcano plot showing $P$ values $(-\log 10)$ versus protein ratio of sLRP2N-E (T3TRAP)-expressing/mocktransfected cells (log2) of 514 secreted and membrane-type proteins $(n=6)$. The horizontal dashed line indicates the $-\log 10$ ( $P$ value) of 1.3 (threshold for statistical significance). The two solid lines (hyperbolas) represent the permutation-based false-discovery rate (FDR) analysis used to evaluate the statistical significance of protein regulation in the two conditions (T3TRAP and controls). Proteins above the hyperbolas are considered statistically significant according to the permutation-based FDR (250 randomizations, FDR: 0.05 , SO: 0.1). (B) Validation of secretome analysis: immunoblots showing the expression of SLRP2N-E (T3TRAP) in the conditioned media of transfected cells, and the subsequent increase in TIMP-3 levels compared to mock-transfected controls. TIMP-1, TIMP-2 and SAPP were detected as proteins non-regulated by T3TRAP expression by the proteomic analysis and validated by Western blots.

\section{DISCUSSION}

TIMP-3 is a major regulator of ECM turnover and TIMP-3-mediated inhibition of metalloproteinases has been shown to have a protective role in different diseases, including cancer, atherosclerosis and arthritis $(9,22,23)$. For this reason there has been considerable interest in understanding the mechanisms controlling TIMP-3 levels in the tissue. We have previously reported that extracellular levels of TIMP-3 can be modulated by LRP-1-mediated endocytosis, which is prevented when the inhibitor is bound to a shed form of LRP-1 or to low molecular weight fragments of the receptor (10). Studies on chondrosarcoma cells and macrophages have shown that ectopic overexpression of Timp3 does not lead to an increase of extracellular levels of the inhibitor, unless its endocytosis is blocked $(11,23)$. Therefore, shedding of LRP-1 and formation of TIMP-3/sLRP-1 complexes seem to be a major mechanism for regulating the bioavailability of TIMP-3 in the tissue. This study aimed to develop a T3TRAP that mimics shed LRP-1 in preventing TIMP-3 from being internalized thereby increasing its levels in the tissue. Therefore, we investigated the LRP-1-TIMP3 interaction in detail in order to find the binding site for TIMP-3 on the receptor and develop a molecule that selectively increases extracellular levels of TIMP-3. 
Table 2. CHANGES IN PROTEIN SECRETION IN T3TRAP EXPRESSING CELLS

Soluble proteins increasing in the conditioned medium of T3TRAP expressing cells

\begin{tabular}{lccccc} 
Protein name & Gene & $I^{a}$ & Ratio $^{b}$ & $p$-value & pept \\
\hline Metalloproteinase inhibitor $\mathbf{3}$ & TIMP3 & P35625 & $\mathbf{4 . 3 5}$ & $\mathbf{1 . 7 6 E - 0 9}$ & $\mathbf{1 7}$ \\
& & & & \\
Chitinase domain-containing protein 1 & CHID1 & Q9BWS & 8.70 & $9.21 \mathrm{E}-09$ & 20 \\
& & 9 & & & \\
Collagen alpha-1(XIV) chain & COL14A1 & Q05707 & 5.65 & $1.36 \mathrm{E}-04$ & 10 \\
Peroxiredoxin-4 & PRDX4 & Q13162 & 2.97 & $6.47 \mathrm{E}-05$ & 25 \\
Laminin subunit beta-2 & LAMB2 & P55268 & 2.21 & $6.10 \mathrm{E}-04$ & 21 \\
Cholinesterase & BCHE & P06276 & 2.03 & $1.79 \mathrm{E}-05$ & 5 \\
\hline
\end{tabular}

Soluble proteins decreasing in the conditioned medium of T3TRAP expressing cells

\begin{tabular}{lccccc} 
Protein name & Gene & ID & Ratio & $p$-value & pept \\
\hline Insulin-like growth factor II & & & & & \\
Apolipoprotein E & IGF2 & P01344 & 0.52 & $1.33 \mathrm{E}-02$ & 5 \\
72 kDa type IV collagenase & APOE & P02649 & 0.56 & $2.40 \mathrm{E}-03$ & 13 \\
Transforming growth factor beta-1 & MMP2 & P08253 & 0.63 & $4.69 \mathrm{E}-04$ & 9 \\
Agrin & TGFB1 & P01137 & 0.67 & $1.10 \mathrm{E}-02$ & 8 \\
\hline
\end{tabular}

\section{Selection of proteins unaltered in T3TRAP expressing cells}

\begin{tabular}{lccccc} 
Protein name & Gene & ID & Ratio & p-value & pept \\
\hline $\begin{array}{l}\text { A disintegrin and metalloproteinase with } \\
\text { thrombospondin motifs 1 }\end{array}$ & ADAMTS1 & Q9UHI8 & 0.99 & $9.14 \mathrm{E}-01$ & 30 \\
Metalloproteinase inhibitor 1 & & & & & \\
Metalloproteinase inhibitor 2 & TIMP1 & P01033 & 0.90 & $8.05 \mathrm{E}-02$ & 12 \\
SPARC & TIMP2 & P16035 & 0.87 & $2.78 \mathrm{E}-01$ & 14 \\
Glia-derived nexin & SPARC & P09486 & 1.07 & $3.97 \mathrm{E}-01$ & 14 \\
Serin protease 23 & SERPINE2 & P07093 & 1.06 & $7.55 \mathrm{E}-01$ & 14 \\
Amyloid precursor protein & PRSS23 & O95084 & 1.10 & $4.20 \mathrm{E}-01$ & 11 \\
\hline
\end{tabular}

Membrane proteins showing reduced shedding in T3TRAP expressing cells

\begin{tabular}{lccccc} 
Protein name & Gene & ID & Ratio & $p$-value & pept \\
\hline Leukocyte surface antigen CD47 & & & & & \\
Integrin beta-1 & CD47 & Q08722 & 0.70 & $1.39 E-03$ & 3 \\
Cell adhesion molecule 1 & ITGB1 & P05556 & 0.71 & $2.06 E-03$ & 15 \\
CD166 antigen & CADM1 & Q9BY67 & 0.71 & $6.28 E-03$ & 8 \\
Cell surface glycoprotein MUC18 & ALCAM & Q13740 & 0.77 & $2.34 \mathrm{E}-03$ & 16 \\
\hline
\end{tabular}

ID: accession number of the protein

Ratio: mean ratio of label-free quantification intensities between T3TRAP-expressing and control conditions $(n=6)$

p-value: for six biological replicates

pept: number of identified peptides of the protein group 
A canonical mode of ligand recognition by LRP-1 underlies a central role for the cysteine-rich complement-type repeats (CRs). These cage-shaped CRs present a characteristic acidic pocket that forms salt bridges with a specific lysine on the ligand moiety, as it was shown for a number of LRP-1 ligands $(24,25)$. Five lysine residues, Lys-26, Lys-27, Lys-30, Lys-76 and Lys-165, are located on the opposite side of the inhibitory ridge of TIMP3 that may be involved in LRP-1 recognition (10). The presence of multiple lysines available for LRP-1 recognition may explain the high affinity of TIMP-3 for LRP-1, with a $\mathrm{K}_{\mathrm{D} \text {,app }}$ in the low nanomolar range, and also the presence of multiple binding sites for TIMP-3 on the receptor. Indeed, the inhibitor efficiently bound all four LRP-1 ligandbinding clusters, with highest affinity for cluster II. The major binding site for TIMP-3 was identified on the $\mathrm{N}$-terminal half of this cluster, but the affinity of the inhibitor for this fragment alone was clearly reduced compared to that for the whole cluster II. We speculate that the majority of the interactions between TIMP-3 and LRP- 1 are mediated by crucial lysines on the inhibitor moiety and CRs on the N-terminal half of cluster II, with additional interactions between TIMP-3 and the C-terminal half that stabilize the binding, thus increasing its affinity.

LRP-1 is currently known to bind over 50 ligands, including a number of TIMP-3 target metalloproteinases (i.e. MMP2, MMP-9, MMP-13, ADAMTS-4 and ADAMTS-5) (13-16). Interestingly, none of these metalloproteinases was able to interact with the N-terminal half of cluster II. This suggests two possibilities: either the binding site for TIMP-3 is physically separated from the binding site of its target metalloproteinases or metalloproteinases require both $\mathrm{N}$-terminal and $\mathrm{C}$-terminal moieties to interact with LRP-1. A secretome analysis of cells expressing a soluble form of the C-terminal half of cluster II did not identify upregulation of metalloproteinases in the conditioned media (not shown), suggesting that both $\mathrm{N}$-and $\mathrm{C}$ terminal halves are required to mediate the interaction between LRP-1 and metalloproteinases. The differential binding of LRP-1 ligands to diverse domains of the receptor may have important physiological consequences. Indeed, different fragments of shed LRP-1 has been found in cancer cells, human brains and cerebrospinal fluids $(26,27)$. Similar fragments were also found associated with extracellular TIMP-3 and shown to inhibit its endocytosis (10). It is interesting to speculate that differential cleavage of LRP-1 can lead to the release of different LRP-1 fragments, thereby specifically preventing the internalization of some proteins (or group of proteins) but not others, thus leading to different biological responses. Determining the precise nature of these fragments may lead to a better understanding of LRP-1 role in ECM turnover, indeed differential cleavage of the receptor can be crucial to regulate the catabolism/anabolism equilibrium of the ECM.

In conclusion, metalloproteinases are known to play important roles in a number of physiological and pathological processes. For its ability to increase tissue levels of TIMP-3, without affecting the turnover of its target metalloproteinases, T3TRAP can potentially be used to investigate the role of TIMP-3 in these processes and exploited in the therapy of diseases characterized by enhanced ECM proteolysis, including arthritis.

\section{EXPERIMENTAL PROCEDURES 4.1 Proteins}

Human recombinant proMMP-2, proMMP-9, proMMP-13 and ADAMTS-5 were purchased from R\&D Systems. BSA was from Sigma-Aldrich. Human midkine from Peptide Institute Inc. The catalytic domain of human MMP-1 (MMP-1 $\Delta \mathrm{C})(28)$, C-terminally FLAG-tagged ADAMTS-4 lacking the C-terminal spacer domain, named ADAMTS4-2 (16), C-terminally FLAG-tagged ADAMTS-5 lacking the Cterminal thrombospondin domain, named ADAMTS5-2, (16), and C-terminally FLAGtagged TIMP-3 (29) were generated as previously described. Human LRP-1 isolated from placenta was purchased from BioMac. His-tagged soluble ligand-binding domains of LRP-1 (sLRP1, sLRP2, sLRP3 and SLRP4) were purified as previously described (16). Briefly, each LRP-1 ligandbinding cluster (sLRP1-4) was transiently expressed in HEK293 cells using TransIT- 
2020 transfection reagent (Mirus). Conditioned media were collected after $72 \mathrm{~h}$ and directly applied to a nickel-nitrilotriacetic acid affinity column (Qiagen). Proteins were then eluted by $500 \mathrm{mM}$ imidazole in HEPES (pH 7.5) buffer containing $150 \mathrm{mM} \mathrm{NaCl}$ and $5 \mathrm{mM} \mathrm{CaCl}_{2}$.

\subsection{Purification of SLRP2N-E-Fc (T3TRAP) \\ The construct for expressing} sLRP2N-E-Fc (T3TRAP) was generated as previously described (30). Briefly, the sequence of human $L R P 1$ corresponding to the amino acid region 826-994 was cloned into pFUSE-Fc (Invitrogen). HEK293 cells were transfected with the construct and after 5 days the conditioned medium was collected and applied onto a protein GSepharose column (GE Healthcare). After washing with $30 \mathrm{ml}$ of PBS, the bound fraction was eluted with $3.6 \mathrm{ml}$ of $100 \mathrm{mM}$ glycine $\mathrm{HCl}(\mathrm{pH} 3.0)$ and neutralized immediately with $0.4 \mathrm{ml}$ of $1.5 \mathrm{M}$ Tris- $\mathrm{HCl}$ (pH 8.8). Purity of the protein was confirmed by SDS-PAGE analysis and subsequent silver staining.

4.3 ELISA for binding of FLAG-tagged TIMP-3, ADAMTS5-2 and ADAMTS4-2 to LRP-1, sLRPs or SLRP2N-E

Full-length LRP-1, purified ligandbinding domains (sLRP1-4) or sLRP2N-E were coated onto an ELISA plate (overnight, $4^{\circ} \mathrm{C}$ ). Wells were blocked with $3 \% \mathrm{BSA}$ in TNC buffer [(50 mM Tris- $\mathrm{HCl}, 150 \mathrm{mM} \mathrm{NaCl}$, $\left.\left.5 \mathrm{mM} \mathrm{CaCl}_{2}, \mathrm{pH} 7.4\right)\left(1 \mathrm{~h}, 37{ }^{\circ} \mathrm{C}\right)\right]$ and washed in TNC buffer containing $0.05 \%$ $(\mathrm{v} / \mathrm{v})$ Brij-35 after this and each subsequent step. Wells were then incubated with various concentrations of FLAG-tagged TIMP-3, ADAMTS4-2 or ADAMTS5-2 in blocking solution [3 $\mathrm{h}$, room temperature (RT)]. Bound protein was detected using anti-FLAG M2 mouse monoclonal antibody $(1 \mathrm{~h}, \mathrm{RT})$ and then with an anti-mouse $\lg \mathrm{G}$ antibody coupled to horseradish peroxidase (Abcam; $1 \mathrm{~h}, \mathrm{RT}$ ). Hydrolysis of tetramethylbenzidine substrate (KPL) was measured at $450 \mathrm{~nm}$ in a plate reader. Each value was normalized by subtracting the amount of ligand bound to control wells that were not coated with LRP-1 or soluble LRP. 1 fragments.

\subsection{Analysis of TIMP-3 accumulation in the conditioned media}

HA tagged minireceptors comprising parts of the ligand-binding cluster II (sLRP2N, sLRP2C, sLRP2N-E and sLRP2C-E, illustrated in Figure 1A) were generated as previously described $(17,30)$. HEK293 cells were transiently transfected with each construct using Fugene HD transfection reagent (Promega) and the conditioned media were collected 5 days after transfection. In order to standardize levels of distinct minireceptors in each conditioned media, their expression was evaluated by Western blot analysis using an anti-HA antibody (Roche) and subsequent band quantification using ImageJ software. Conditioned media containing standardized amount of each minireceptor were incubated with a number of different cell lines (including U251 human glioblastoma, PC3 prostate adenocarcinoma and COS1) for $24 \mathrm{~h}$, and accumulation of TIMP-3 in the conditioned media was evaluated by immunoblotting using a polyclonal antiTIMP-3 antibody (Millipore). No TIMP-3 was detected by Western blot in the minireceptor-containing conditioned media before incubation with cell lines. Negative controls were incubated with conditioned media collected from cells transfected with an empty vector (pcDNA3.1, Invitrogen). Positive controls were incubated in conditioned medium from mock transfected cells, supplemented with $200 \mu \mathrm{g} / \mathrm{ml}$ heparin that blocks TIMP-3 endocytosis $(10,11)$. Pixel density of the bands corresponding to TIMP-3 was quantified using ImageJ and displayed as a percentage of the values corresponding to TIMP-3 bands in the heparin-treated samples (positive controls).

\subsection{Analysis of TIMP-3 endocytosis}

Purified TIMP-3-FLAG (5 nM) was incubated with an equimolar concentration of purified SLRP2N-E for $1 \mathrm{~h}$ at $37{ }^{\circ} \mathrm{C}$, in order to form TIMP-3/sLRP2N-E complexes in DMEM with $0.1 \%$ of serum. Then, complexes were added onto U251 cells and conditioned media collected at different time points. TIMP-3-FLAG in the conditioned media was detected by Western blotting, using an anti-FLAG M2 antibody. Amount of TIMP-3-FLAG in different samples was determined by band quantification and 
plotted as a percentage of values corresponding to the amount of TIMP-3 at 0 h.

\subsection{Analysis of TIMP-3 and metalloproteinase binding to sLRP2N-E by surface plasmon resonance (SPR)}

The binding of TIMP-3 and metalloproteinases to the SLRP2N-E was analyzed by Biacore 3000. Purified sLRP2N-E was immobilized on a CM5 sensor chip by amine coupling using $\mathrm{N}$ hydroxysuccinimide (NHS). Recombinant TIMP-3, midkine, proMMP-2, proMMP-9 and proMMP-13 and ADAMTS-5 were injected over the immobilized SLRP2N-E at eight concentrations ranging from 0 to $80 \mathrm{nM}$, unless differently specified. Each experiment was performed in triplicate.

4.7 Proteomic analysis of conditioned media from T3-TRAP expressing HEK cells

HEK cells were cultured in 6-well plates and transfected with SLRP2N-E or empty vector in serum-free DMEM. After 24 $h$, supernatants were harvested and centrifuged to remove cell debris. Protein concentration in supernatants was measured using the colorimetric $660 \mathrm{~nm}$ assay (Thermo Fisher Scientific, US). A protein amount of $25 \mathrm{\mu g}$ per sample was subjected to proteolytic digestion using the filter assisted sample preparation (FASP) protocol with $10 \mathrm{kDa}$ Vivacon spin filters (Sartorius, Germany) (31). Briefly, proteins were denatured with $8 \mathrm{M}$ urea in $100 \mathrm{mM}$ $\mathrm{TRIS} / \mathrm{HCl} \mathrm{pH} 8.5$, followed by reduction with $100 \mathrm{mM}$ dithiotreitol for $30 \mathrm{~min}$ and subsequent alkylation with $50 \mathrm{mM}$ iodoacetamide for $30 \mathrm{~min}$ in the dark. Proteins were digested with $0.5 \mu \mathrm{g}$ LysC (Promega) over night and subsequently with $0.5 \mu \mathrm{g}$ trypsin (Promega) for $4 \mathrm{~h}$ at $37^{\circ} \mathrm{C}$. Peptides were desalted on reversed phase C18 StageTips, and eluted using $40 \mu \mathrm{l}$ of $60 \%$ acetonitrile in $0.1 \%$ formic acid. The volume was reduced in a SpeedVac and the peptides were resuspended in $0.1 \%$ formic acid. Proteolytic peptides were desalted by stop and go extraction (STAGE) with C18 tips (32). The purified peptides were dried by vacuum centrifugation. Samples were dissolved in $40 \mu \mathrm{L} 0.1 \%$ formic acid and analyzed by LC-MS/MS.
A number of proteins detected in the analysis were further validated by immunoblotting (T3TRAP, TIMP-3, TIMP-1, TIMP-2, sAPP).

\subsection{Proeteomic data analysis}

The data was analyzed by the software Maxquant (maxquant.org, MaxPlanckInstitute Munich) version 1.5.2.8. The MS data was searched against a reviewed canonical fasta database of Homo sapiens from UniProt (download: December $12^{\text {th }}$ 2014, 16685 entries). Trypsin was defined as protease. Two missed cleavages were allowed for the database search. The option first search was used to recalibrate the peptide masses within a window of $20 \mathrm{ppm}$. For the main search peptide and peptide fragment mass tolerances were set to 4.5 and $20 \mathrm{ppm}, \quad$ respectively. Carbamidomethylation of cysteine was defined as static modification. Acetylation of the protein $\mathrm{N}$-term as well as oxidation of methionine was set as variable modifications. The false discovery rate for both peptides and proteins was adjusted to less than $1 \%$. Label free quantification (LFQ) of proteins required at least two ratio counts of unique peptides. Only unique peptides were used for quantification. The LFQ values were log2 transformed and a two sided heteroscedastic student's ttest was used to evaluate statistically significant regulated proteins between mocktransfected and T3TRAP-expressing HEK293 cells. A P value less than $5 \%$ was set as significance threshold. Permutation-based falsediscovery rate (FDR) analysis was used to evaluate the statistical significance of protein regulation in the two conditions (250 randomizations, FDR: 0.05, SO: 0.1).

\section{AUTHOR CONTRIBUTION}

SDS conceived and coordinated the study, performed and analyzed experiments, and wrote the paper. $\mathrm{KY}$ performed experiments showed in Figures $1 \mathrm{~B}$ and 4B. MP performed experiments shown in 5B. KS provided technical assistance and contributed to design experiments. SAM provided technical assistance and contributed to analyze the experiments shown in figure 5A. AP provided technical assistance and contributed to write the manuscript. SFL contributed to design and 
analyze the experiments of proteomics. LT contributed to analyze the experiments and write the manuscript. $\mathrm{HN}$ contributed to design the study, analyze the experiments and write the manuscript. KK contributed to design and analyze the experiments, and to write the manuscript

\section{DECLARATION OF INTEREST}

The authors declare that they have no conflicts of interest with the content of this article

\section{ACKNOWLEDGEMENTS}

SDS was supported by the Japan Society for Promotion of Science (JSPS) short-term fellowship, the Marie Sklodovska-Curie individual fellowship and Technical University Munich Foundation Fellowship (TUFF). KY was supported by grants from the Arthritis Research UK (20563). Work in the laboratory of SFL has been funded through grants of the DFG (SyNergy and FOR2290); BMBF (JPND-RiModFTD), the Belgian Agency for Innovation by Science and Technology (IWT) and the Alzheimer Research Price of the Breuer Foundation. KY was supported by grants from the Arthritis Research UK (20563).

We would like to thank Prof. Carl
Blobel, Prof. Gillian Murphy and Prof. Athanasios Papadopoulos for their critical reading of the manuscript and useful comments, and Shoma Tsubota and Dr. Kenji Yuchimura for technical assistance.

\section{KEYWORDS}

Tissue inhibitor of metalloproteinase- 3 low-density lipoprotein receptor-related protein-1 matrix metalloproteinases

ADAMs ADAMTSs extracellular matrix inflammation

\section{Abbreviations used:}

TIMP-3, tissue inhibitor of metalloproteinases-3; LRP-1, low density lipoprotein receptor-related protein-1; MMPs, matrix metalloproteinases; ADAMs, adamalysin-like metalloproteinases; ADAMTSs, adamalysin-like metalloproteinases with thrombospondin motifs; TNFa, tumor necrosis factor alpha; $\mathrm{OA}$, osteoarthritis; RA, rheumatoid arthritis; ECM, extracellular matrix; EGF, epidermal growth factor; CRs, cysteine-rich complement-type ligands. 


\section{REFERENCES}

1. Nagase, H., Visse, R., and Murphy, G. (2006) Structure and function of matrix metalloproteinases and TIMPs. Cardiovasc Res 69, 562-573

2. Becherer, J. D., and Blobel, C. P. (2003) Biochemical properties and functions of membrane-anchored metalloprotease-disintegrin proteins (ADAMs). Curr Top Dev Biol 54, 101-123

3. Brew, K., and Nagase, H. (2010) The tissue inhibitors of metalloproteinases (TIMPs): an ancient family with structural and functional diversity. Biochim Biophys Acta 1803, 55-71

4. Kassiri, Z., Oudit, G. Y., Kandalam, V., Awad, A., Wang, X., Ziou, X., Maeda, N., Herzenberg, A. M., and Scholey, J. W. (2009) Loss of TIMP3 enhances interstitial nephritis and fibrosis. J Am Soc Nephrol 20, 1223-1235

5. Leco, K. J., Waterhouse, P., Sanchez, O. H., Gowing, K. L., Poole, A. R., Wakeham, A., Mak, T. W., and Khokha, R. (2001) Spontaneous air space enlargement in the lungs of mice lacking tissue inhibitor of metalloproteinases-3 (TIMP-3). J Clin Invest 108, 817-829

6. Fata, J. E., Leco, K. J., Voura, E. B., Yu, H. Y., Waterhouse, P., Murphy, G., Moorehead, R. A., and Khokha, R. (2001) Accelerated apoptosis in the Timp3-deficient mammary gland. J. Clin. Invest. 108, 831-841

7. Mohammed, F. F., Smookler, D. S., Taylor, S. E., Fingleton, B., Kassiri, Z., Sanchez, O. H., English, J. L., Matrisian, L. M., Au, B., Yeh, W. C., and Khokha, R. (2004) Abnormal TNF activity in Timp3-/- mice leads to chronic hepatic inflammation and failure of liver regeneration. Nat Genet 36, 969-977

8. Gendron, C., Kashiwagi, M., Hughes, C., Caterson, B., and Nagase, H. (2003) TIMP-3 inhibits aggrecanase-mediated glycosaminoglycan release from cartilage explants stimulated by catabolic factors. FEBS Lett. 555, 431436

9. $\quad$ Black R.A., C. B., Slack J., Tocker J., Eisenman J., Jacobson E., Delaney J., Winters D., Hecht R., Bendele A. (2006) Injected TIMP-3 protects cartilage in a rat meniscal tear model. Osteoarthritis and Cartilage

10. Scilabra, S. D., Troeberg, L., Yamamoto, K., Emonard, H., Thogersen, I., Enghild, J. J., Strickland, D. K., and Nagase, H. (2013) Differential regulation of extracellular tissue inhibitor of metalloproteinases-3 levels by cell membrane-bound and shed low density lipoprotein receptor-related protein 1. J Biol Chem 288, 332-342

11. Troeberg, L., Fushimi, K., Khokha, R., Emonard, H., Ghosh, P., and Nagase, $\mathrm{H}$. (2008) Calcium pentosan polysulfate is a multifaceted exosite inhibitor of aggrecanases. FASEB J. 22, 3515-3524

12. Lillis, A. P., Van Duyn, L. B., Murphy-Ullrich, J. E., and Strickland, D. K. (2008) LDL receptor-related protein 1: unique tissue-specific functions revealed by selective gene knockout studies. Physiol Rev 88, 887-918

13. Barmina, O. Y., Walling, H. W., Fiacco, G. J., Freije, J. M., Lopez-Otin, C., Jeffrey, J. J., and Partridge, N. C. (1999) Collagenase-3 binds to a specific receptor and requires the low density lipoprotein receptor-related protein for internalization. J Biol Chem 274, 30087-30093

14. Emonard, H., Bellon, G., Troeberg, L., Berton, A., Robinet, A., Henriet, P., Marbaix, E., Kirkegaard, K., Patthy, L., Eeckhout, Y., Nagase, H., Hornebeck, W., and Courtoy, P. J. (2004) Low density lipoprotein receptor-related protein mediates endocytic clearance of pro-MMP-2.TIMP-2 complex through a thrombospondin-independent mechanism. J Biol Chem 279, 54944-54951

15. Hahn-Dantona, E., Ruiz, J. F., Bornstein, P., and Strickland, D. K. (2001) The low density lipoprotein receptor-related protein modulates levels of matrix metalloproteinase 9 (MMP-9) by mediating its cellular catabolism. J Biol Chem 276, 15498-15503 
16. Yamamoto, K., Owen, K., Parker, A. E., Scilabra, S. D., Dudhia, J., Strickland, D. K., Troeberg, L., and Nagase, H. (2014) Low density lipoprotein receptorrelated protein 1 (LRP1)-mediated endocytic clearance of a disintegrin and metalloproteinase with thrombospondin motifs-4 (ADAMTS-4): functional differences of non-catalytic domains of ADAMTS-4 and ADAMTS-5 in LRP1 binding. J Biol Chem 289, 6462-6474

17. Chen, S., Bu, G., Takei, Y., Sakamoto, K., Ikematsu, S., Muramatsu, T., and Kadomatsu, K. (2007) Midkine and LDL-receptor-related protein 1 contribute to the anchorage-independent cell growth of cancer cells. J Cell Sci 120, 4009-4015

18. Estrella, C., Rocks, N., Paulissen, G., Quesada-Calvo, F., Noel, A., Vilain, E., Lassalle, P., Tillie-Leblond, I., Cataldo, D., and Gosset, P. (2009) Role of A disintegrin and metalloprotease-12 in neutrophil recruitment induced by airway epithelium. Am J Respir Cell Mol Biol 41, 449-458

19. Shastry, S., and Tyagi, S. C. (2004) Homocysteine induces metalloproteinase and shedding of beta-1 integrin in microvessel endothelial cells. J Cell Biochem 93, 207-213

20. Nagara, Y., Hagiyama, M., Hatano, N., Futai, E., Suo, S., Takaoka, Y., Murakami, Y., Ito, A., and Ishiura, S. (2012) Tumor suppressor cell adhesion molecule 1 (CADM1) is cleaved by a disintegrin and metalloprotease 10 (ADAM10) and subsequently cleaved by gamma-secretase complex. Biochem Biophys Res Commun 417, 462-467

21. Rosso, O., Piazza, T., Bongarzone, I., Rossello, A., Mezzanzanica, D., Canevari, S., Orengo, A. M., Puppo, A., Ferrini, S., and Fabbi, M. (2007) The ALCAM shedding by the metalloprotease ADAM17/TACE is involved in motility of ovarian carcinoma cells. Mol Cancer Res 5, 1246-1253

22. Baker, A. H., George, S. J., Zaltsman, A. B., Murphy, G., and Newby, A. C. (1999) Inhibition of invasion and induction of apoptotic cell death of cancer cell lines by overexpression of TIMP-3. Br J Cancer 79, 1347-1355

23. Casagrande, V., Menghini, R., Menini, S., Marino, A., Marchetti, V., Cavalera, M., Fabrizi, M., Hribal, M. L., Pugliese, G., Gentileschi, P., Schillaci, O., Porzio, O., Lauro, D., Sbraccia, P., Lauro, R., and Federici, M. (2012) Overexpression of tissue inhibitor of metalloproteinase 3 in macrophages reduces atherosclerosis in low-density lipoprotein receptor knockout mice. Arterioscler., Thromb., Vasc. Biol. 32, 74-81

24. Strickland, D. K., Au, D. T., Cunfer, P., and Muratoglu, S. C. (2014) Lowdensity lipoprotein receptor-related protein-1: role in the regulation of vascular integrity. Arterioscler Thromb Vasc Biol 34, 487-498

25. van den Biggelaar, M., Sellink, E., Klein Gebbinck, J. W., Mertens, K., and Meijer, A. B. (2011) A single lysine of the two-lysine recognition motif of the D3 domain of receptor-associated protein is sufficient to mediate endocytosis by low-density lipoprotein receptor-related protein. Int J Biochem Cell Biol 43, 431-440

26. Liu, Q., Zhang, J., Tran, H., Verbeek, M. M., Reiss, K., Estus, S., and Bu, G. (2009) LRP1 shedding in human brain: roles of ADAM10 and ADAM17. Mol Neurodegener 4, 17

27. Rozanov, D. V., Hahn-Dantona, E., Strickland, D. K., and Strongin, A. Y. (2004) The low density lipoprotein receptor-related protein LRP is regulated by membrane type-1 matrix metalloproteinase (MT1-MMP) proteolysis in malignant cells. J. Biol. Chem. 279, 4260-4268

28. Chung, L., Shimokawa, K., Dinakarpandian, D., Grams, F., Fields, G. B., and Nagase, H. (2000) Identification of the (183)RWTNNFREY(191) region as a critical segment of matrix metalloproteinase 1 for the expression of collagenolytic activity. J Biol Chem 275, 29610-29617 
29. Troeberg, L., Fushimi, K., Scilabra, S. D., Nakamura, H., Dive, V., Thogersen, I. B., Enghild, J. J., and Nagase, H. (2009) The C-terminal domains of ADAMTS-4 and ADAMTS-5 promote association with N-TIMP-3. Matrix Biol 28, 463-469

30. Sakamoto, K., Bu, G., Chen, S., Takei, Y., Hibi, K., Kodera, Y., McCormick, L. M., Nakao, A., Noda, M., Muramatsu, T., and Kadomatsu, K. (2011) Premature ligand-receptor interaction during biosynthesis limits the production of growth factor midkine and its receptor LDL receptor-related protein 1. J Biol Chem 286, 8405-8413

31. Wisniewski, J. R., Zougman, A., Nagaraj, N., and Mann, M. (2009) Universal sample preparation method for proteome analysis. Nat Methods 6, 359-362

32. Rappsilber, J., Ishihama, Y., and Mann, M. (2003) Stop and go extraction tips for matrix-assisted laser desorption/ionization, nanoelectrospray, and LC/MS sample pretreatment in proteomics. Anal Chem 75, 663-670 